Nuclear Physics B205 [FS5] (1982) 285-300

(C) North-Holland Publishing Company

\title{
SU(N) GAUGE THEORY COUPLINGS ON ASYMMETRIC LATTICES
}

\author{
Frithjof KARSCH \\ Fakultät für Physik, Universität Bielefeld, Germany
}

Received 29 December 1981

\begin{abstract}
The connection between euclidean and hamiltonian lattice QCD requires the use of asymmetric lattices, which in turn implies the necessity of two coupling parameters. We analyse the dependence of space- and time-like couplings $g_{\sigma}$ and $g_{\tau}$ on the different lattice spacings $a$ and $a_{\tau}$ in space and time directions. Using the background field method we determine the derivatives of the couplings with respect to the asymmetry factor $\xi=a / a_{\tau}$ in the weak coupling limit, obtaining for $\xi=1$ the values

$$
\begin{aligned}
\left(\partial g_{\sigma}^{-2} / \partial \xi\right)_{\xi=1} & = \begin{cases}0.11403, & N=2, \\
0.20161, & N=3,\end{cases} \\
\left(\partial g_{\tau}^{-2} / \partial \xi\right)_{\xi=1} & = \begin{cases}-0.06759, & N=2, \\
-0.13195, & N=3 .\end{cases}
\end{aligned}
$$

We argue that the sum of these derivatives has to be equal to $b_{0}=11 N / 48 \pi^{2}$ and determine the $A$ parameter for asymmetric lattices. In the limit $\xi \rightarrow \infty$ all our results agree with those of $A$. and $P$. Hasenfratz.
\end{abstract}

\section{Introduction}

During the past year much progress has been made in extracting physically interesting quantities out of Monte Carlo simulations of non-abelian gauge theories on the lattice [1-9]. In order to assign absolute values to dimensional quantities like the string tension [1-3], glueball mass [3-5] or the critical temperature of the gluon gas [6-9], it is necessary to know the connection between the lattice and continuum scale parameters $\Lambda_{\mathrm{L}} / \Lambda_{\text {MOM }}[10,11]$. This ratio has by now been calculated for different lattice actions [12], including also the contribution of fermions [13] on a four-dimensional euclidean lattice. However, the euclidean lattice with equal lattice spacings, $a_{\mu}=a, \mu=1,2,3,4$, in all four space-time directions, is just one way of introducing a lattice cut-off for the continuum theory. Another possibility is the hamiltonian version of a lattice cut-off, with $a_{\mu}=a$ for $\mu=1,2$, 3 and $a_{4}=0$. Of course, the scale parameters depend on the special lattice regularization scheme chosen, and the relation between $\Lambda_{\mathrm{E}}\left(a_{4}=a\right)$ and $\Lambda_{\mathrm{H}}\left(a_{4}=0\right)$ has been calculated recently by $A$. and P. Hasenfratz [14].

In the case of finite-temperature calculations on the lattice, the introduction of different scales in space and time ( $\simeq$ inverse temperature) directions follows quite naturally $[7,8]$. For simplicity, one uses equal lattice spacings in the three space 
directions $a_{i}=a$ and an independent spacing in the time direction, $a_{4}=a_{\tau}$. These asymmetric lattices are characterized by the asymmetry factor $\xi=a / a_{\tau}$. For $\xi=1$ one recovers the euclidean formulation, while the limit $\xi \rightarrow \infty$ yields the hamiltonian version of lattice gauge theories.

For pure $\mathrm{SU}(N)$ gauge systems the partition function on an asymmetric lattice is given by

$$
Z\left(N_{\sigma}, N_{\tau}, a, \xi\right)=\int \prod_{x, \mu} \mathrm{d} U_{x, x+\mu} \mathrm{e}^{-S(U)},
$$

where $N_{\sigma}, N_{\tau}$ denote the number of lattice sites in space and time directions. The lattice action becomes

$$
S(U)=\beta_{\sigma} \sum_{x} \sum_{\substack{i>j \\ i \neq 4}} P_{i j}+\beta_{\tau} \sum_{x} \sum_{i \neq 4} P_{4 i},
$$

with the plaquette terms

$$
P_{\mu \nu}=\operatorname{Tr}\left(1-U_{x, x+\mu} U_{x+\mu, x+\mu+\nu} U_{x+\nu, x+\mu+\nu}^{+} U_{x, x+\nu}^{+}\right)+\text {h.c . }
$$

The two couplings in eq. (1.2) are necessary in order to keep physics unchanged under independent variations of $a$ and $\xi$. They are parametrized as

$$
\beta_{\sigma}=\frac{1}{g_{\sigma}^{2}(a, \xi)} \xi^{-1}, \quad \beta_{\tau}=\frac{1}{g_{\tau}^{2}(a, \xi)} \xi,
$$

where the additional factors of $\xi$ in eq. (1.4) are chosen such that the naive continuum limit of eq. (1.2) gives back the classical action

$$
S(U) \underset{\substack{a \rightarrow 0 \\ \xi \text { fixed }}}{\longrightarrow} \int F_{\mu \nu}^{b}(x) F_{\mu \nu}^{b}(x) \mathrm{d}^{4} x .
$$

In this way the $\xi$-dependence of the couplings $g_{\sigma}$ and $g_{\tau}$ is due to quantum corrections, which survive the continuum limit.

At finite temperature eq. (1.1) describes the thermodynamics of an interacting gluon gas in the volume $V=\left(N_{\sigma} a\right)^{3}$ with the inverse temperature given by $\beta=$ $N_{\tau} a / \xi$. From eq. (1.1) one can derive all thermodynamic quantities by taking derivatives with respect to $a$ or $\xi$. For instance the energy density of a gluon gas [8] (including still the vacuum energy density) is given by

$$
\varepsilon a^{4}=-\xi^{2}\left[\left(\partial \beta_{\sigma} / \partial \xi\right) \sum_{\substack{i>i \\ i \neq 4}}\left\langle P_{i j}\right\rangle+\left(\partial \beta_{\tau} / \partial \xi\right) \sum_{i \neq 4}\left\langle P_{4 i}\right\rangle\right] .
$$

Thus it is essential for the calculation of $\varepsilon a^{4}$ and other thermodynamic quantities to know the $\xi$-dependence of the couplings $g_{\sigma}$ and $g_{\tau}$ in order to get the contribution from $\left(\partial g_{\sigma(\tau)}^{-2} / \partial \xi\right)$ to the derivatives of $\beta_{\sigma}$ and $\beta_{\tau}$ in eq. (1.6). As finite-temperature Monte Carlo simulations for the energy density have in general been performed 
on a euclidean lattice with $\xi=1[8,15]$, we are in particular interested in the value of the derivatives of the couplings for $\xi=1$. From first Monte Carlo calculations it was expected that the quantum corrections $\left(\partial g_{\sigma(\tau)}^{-2} / \partial \xi\right)_{\xi=1}$ to the derivatives are small [8], and recently a more precise Monte Carlo evaluation of the SU(3) couplings supported this [15].

In the weak coupling limit of $\mathrm{SU}(N)$ lattice gauge theory, the dependence of $g_{\sigma}$ and $g_{\tau}$ on the asymmetry factor $\xi$ can be determined analytically from the effective action, using the background field method on the lattice [11], and this is the main objective of the present paper. In sect. 2 we will calculate the derivatives of the couplings with respect to $\xi$ as well as the $\xi$-dependent lattice scale parameter $A(\xi)$. In sect. 3 we discuss, how one can use the invariance of the string tension under changes of $\xi$ to obtain the "sum rule"

$$
\left(\partial g_{\sigma}^{-2} / \partial \xi\right)_{\xi=1}+\left(\partial g_{\tau}^{-2} / \partial \xi\right)_{\xi=1}=11 N / 48 \pi^{2}
$$

for the derivatives of the couplings in the continuum limit and to get an idea of their strong coupling behaviour. Sect. 4 contains our conclusions. Appendix A summarizes some integrals necessary for the calculation of the effective action and in appendix B we give a 12th order strong coupling expansion of the string tension on asymmetric lattices.

\section{The continuum limit for asymmetric lattices}

In the continuum limit, physical quantities must become independent of the kind of lattice regularization chosen. In the case of an asymmetric lattice with different lattice spacings $a$ and $a_{\tau}$ for space and time directions, this means that such quantities have to be independent of the asymmetry factor $\xi=a / a_{\tau}$. In order to achieve this we have to introduce two couplings in the lattice action (1.2), which both depend on $\xi$. For $\xi=1$, we get back the usual euclidean formulation of a lattice gauge theory, where both couplings are equal,

$$
g_{\sigma}^{-2}(a, 1)=g_{\tau}^{-2}(a, 1) \equiv g_{\mathrm{E}}^{-2}(a) .
$$

For pure $\mathrm{SU}(N)$ gauge theories on the lattice the behaviour of the bare euclidean coupling $g_{\mathrm{E}}(a)$ in the continuum limit $a \rightarrow 0$ is related to the lattice spacing $a$ through the renormalization group relation

$$
a \Lambda_{\mathrm{E}}=\left(b_{0} g_{\mathrm{E}}^{2}\right)^{-b_{1} /\left(2 b_{0}^{2}\right)} \exp \left\{-1 /\left(2 b_{0} g_{\mathrm{E}}^{2}\right)\right\} .
$$

Here $A_{\mathrm{E}}$ is the lattice scale parameter for the symmetric lattice and $b_{0}, b_{1}$ are the first two coefficients in the Callan-Symanzik equation

$$
b_{0}=\frac{11 N}{48 \pi^{2}}, \quad b_{1}=\frac{34}{3}\left(\frac{N}{16 \pi^{2}}\right)^{2} .
$$


When we now go over to an asymmetric lattice, $\xi \neq 1$, the couplings $g_{\sigma}^{-2}$ and $g_{\tau}^{-2}$ are no longer equal; but in the weak coupling limit they still can be expanded in terms of the symmetric value $g_{\mathrm{E}}^{-2}[14]$,

$$
\begin{aligned}
& g_{\sigma}^{-2}(a, \xi)=g_{\mathrm{E}}^{-2}(a)+c_{\sigma}(\xi)+\mathrm{O}\left(g_{\mathrm{E}}^{2}\right), \\
& g_{\tau}^{-2}(a, \xi)=g_{\mathrm{E}}^{-2}(a)+c_{\tau}(\xi)+\mathrm{O}\left(g_{\mathrm{E}}^{2}\right) .
\end{aligned}
$$

As a consequence of this change of the couplings also the lattice scale parameter becomes $\xi$ dependent. To see this more clearly we may write the action (1.2) in the form

$$
S(U)=\frac{1}{g_{\xi}^{2}}\left[\eta \xi^{-1} \sum_{\substack { x \\
\begin{subarray}{c}{i>i \\
i \neq 4{ x \\
\begin{subarray} { c } { i > i \\
i \neq 4 } }\end{subarray}} P_{i j}+\eta^{-1} \xi \sum_{x} \sum_{i \neq 4} P_{4 i}\right]
$$

where we have introduced two new couplings $g_{\xi}^{2}=g_{\sigma} \cdot g_{\tau}$ and $\eta=\left(g_{\tau}^{2} / g_{\sigma}^{2}\right)^{1 / 2}$. Due to eq. (2.4) $\eta$ approaches 1 in the continuum limit $(a \rightarrow 0, \xi$ fixed), so that the action depends again only on one coupling $g_{\xi}$ in this limit. The relation between $g_{\xi}$ and the lattice spacing $a$ is then again given by the renormalization group equation (2.2) with $\Lambda_{\mathrm{E}}$ replaced by $\Lambda(\xi)$ and $g_{\mathrm{E}}$ replaced by $g_{\xi}$. Therefore, one gets for the ratio of the two scale parameters

$$
\Lambda(\xi) / \Lambda_{\mathrm{E}}=\exp \left\{-\left(c_{\sigma}(\xi)+c_{\tau}(\xi)\right) / 4 b_{0}\right\} .
$$

In the hamiltonian limit $\xi \rightarrow \infty$ this ratio as well as the functions $c_{\sigma}(\xi)$ and $c_{\tau}(\xi)$ have been determined recently by A. and P. Hasenfratz [14]. We will perform the corresponding calculations for arbitrary $\xi$, in order to obtain in addition also the derivatives of the couplings,

$$
\left(\partial g_{\sigma(\tau)}^{-2} / \partial \xi\right)=\left(\partial c_{\sigma(\tau)} / \partial \xi\right)+\mathrm{O}\left(g_{\mathrm{E}}^{2}\right),
$$

which, as we saw, are of particular interest for the calculation of thermodynamic quantities.

The functions $c_{\sigma}(\xi)$ and $c_{\tau}(\xi)$ can be found by calculating the effective action $S_{\text {eff }}^{(\xi)}$ of the $\mathrm{SU}(N)$ lattice gauge theory for two different lattice regularization procedures, i.e., for two different lattice cut-offs such as $\xi=1$ and some $\xi \neq 1$. The condition that in the continuum limit the effective action has to be independent of the regularization chosen,

$$
\Delta S_{\text {eff }}=S_{\text {eff }}^{(\xi=1)}-S_{\text {eff }}^{(\xi \neq 1)}=0,
$$

leads to eq. (2.4) with determined functions $c_{\sigma}(\xi)$ and $c_{\tau}(\xi)$. The effective action can be most easily evaluated by using the background field method on the lattice [11]. The basic concepts of this method have been discussed in detail elsewhere $[11,12]$. We thus will concentrate on the specific notations necessary for the calculations on asymmetric lattices. 
The SU(N) gauge field variables $U_{x, x+\mu}$ appearing in the action (1.2), (1.3) are parametrized in terms of a classical background field $B_{\mu}(x)$ and of a quantum field $\alpha_{\mu}(x)$

$$
U_{x, x+\mu}=\mathrm{e}^{i \mathrm{~g}_{\mu} a_{\mu} \alpha_{\mu}(x)} U_{x, x+\mu}^{(0)}
$$

with

$$
U_{x, x+\mu}^{(0)}=\mathrm{e}^{i a_{\mu} B_{\mu}(x)} .
$$

For the couplings we have $g_{\mu}=g_{\sigma}$ for $\mu=1,2,3$ and $g_{4}=g_{r}$. The fields can be expanded in terms of the group generators $\lambda^{a}, a=1, \ldots, N^{2}-1$,

$$
B_{\mu}=B_{\mu}^{a} \lambda^{a} / 2, \quad \alpha_{\mu}=\alpha_{\mu}^{a} \lambda^{a} / 2,
$$

where the generators are normalized so that $\operatorname{Tr} \lambda^{a} \lambda^{b}=2 \delta_{a b}$. In addition we define covariant derivatives as

$$
\begin{aligned}
& D_{\mu} f(x)=\frac{1}{a_{\mu}}\left(U_{x, x+\mu} f(x+\mu) U_{x, x+\mu}^{+}-f(x)\right), \\
& \overline{D_{\mu}} f(x)=\frac{1}{a_{\mu}}\left(U_{x-\mu, x}^{+} f(x-\mu) U_{x-\mu, x}-f(x)\right) .
\end{aligned}
$$

The lattice derivatives $\Delta_{\mu} f(x)$ and $\overline{\Delta_{\mu}} f(x)$ follow from eq. (2.12) by setting $U \equiv \mathbb{1}$, and we write $D_{\mu}^{(0)}, \overline{D_{\mu}^{(0)}}$, whenever we replace $U_{x, x+\mu}$ by $U_{x, x+\mu}^{(0)}$.

Now we can use the parametrization (2.9) of the $\mathrm{SU}(N)$ link variables to expand the action (1.2) up to terms quadratic in the gauge fields $\alpha_{\mu}$. This is enough for the calculation of the quantum corrections to the $\mathrm{SU}(N)$ couplings in the weak coupling limit. Notice that up to this order $g_{\tau}^{2} / g_{\mathrm{E}}^{2}=g_{\sigma}^{2} / g_{\mathrm{E}}^{2}=1$. In the weak coupling limit we have to add the gauge-fixing term

$$
S_{\mathrm{gf}}=a^{3} a_{\tau} \sum_{x} \operatorname{tr}\left(\sum_{\mu} \overline{D_{\mu}^{(0)}} \alpha_{\mu}(x)\right)^{2},
$$

and the resulting ghost term

$$
S_{\mathrm{gh}}=2 a^{3} a_{\tau} \sum_{x} \sum_{\mu} \operatorname{tr}\left[\left(D_{\mu}^{(0)} \phi(x)\right)^{+}\left(D_{\mu}^{(0)} \phi(x)\right)\right]
$$

to the action (1.2). The expansion of the action now becomes

$$
S(U)=S\left(U^{(0)}\right)+S_{2}(\alpha, \phi, B),
$$

with

$$
S_{2}(\alpha, \phi, B)=S_{\mathrm{sc}}+S_{\mathrm{T}}+S_{\mathrm{A}}+S_{B}+S_{\mathrm{gh}} .
$$

The different contributions to $S_{2}$ are given by

$$
S_{\mathrm{sc}}=a^{3} a_{\tau} \sum_{x} \sum_{\mu, \nu} \operatorname{tr}\left[\left(D_{\mu}^{(0)} \alpha_{\nu}\right)\left(D_{\mu}^{(0)} \alpha_{\nu}\right)\right]
$$




$$
\begin{gathered}
S_{\mathrm{T}}=-\frac{a^{3} a_{\tau}}{16 N} \sum_{x} \sum_{\substack{\mu, \nu \\
a, b}}\left(a_{\mu} a_{\nu}\right)^{2}\left(\Delta_{\mu} \alpha_{\nu}^{a}-\Delta_{\nu} \alpha_{\nu}^{a}\right)^{2} F_{\mu \nu}^{b}(x) F_{\mu \nu}^{b}(x) ; \\
S_{A}=\frac{1}{2} a^{3} a_{\tau} \sum_{x} \sum_{\mu, \nu} \operatorname{tr}\left(A_{\mu \nu}(x) F_{\mu \nu}(x)\right),
\end{gathered}
$$

where

$A_{\mu \nu}(x)=-2 i\left\{2\left[\alpha_{\nu}, \alpha_{\mu}\right]+a_{\nu}\left[\alpha_{\nu}, D_{\nu}^{(0)} \alpha_{\mu}\right]+a_{\mu}\left[D_{\mu}^{(0)} \alpha_{\nu}, \alpha_{\mu}\right]-\frac{1}{2} a_{\mu} a_{\nu}\left[D_{\nu}^{(0)} \alpha_{\mu}, D_{\mu}^{(0)} \alpha_{\nu}\right]\right\}$

$$
S_{B}=\frac{1}{2} a^{3} a_{\tau} \sum_{x} \sum_{\mu, \nu} \operatorname{tr}\left(B_{\mu \nu}(x) F_{\mu \nu}(x)\right)
$$

where

$$
B_{\mu \nu}(x)=-i\left(a_{\mu}\left[D_{\nu}^{(0)} \alpha^{\mu}, \alpha^{\mu}\right]+a_{\nu}\left[\alpha^{\nu}, D_{\mu}^{(0)} \alpha^{\nu}\right]\right)
$$

and the ghost field term $S_{\text {gh }}$ is given by eq. (2.13). The field-strength tensor $F_{\mu \nu}(x)$ is built up from the background fields $B_{\mu}(x)$ in the usual way. Actually there is an additional term $S_{\mathrm{T}}^{\prime}$ [14] contributing to $S_{2}$, but as this term is of odd order in the Lorentz indices of the gauge fields $\alpha_{\mu}$, it cannot contribute to the expansion for the effective action. Therefore, we have omitted it here.

Expanding also the integration measure $\prod_{x, \mu} \mathrm{d} U_{x, x+\mu}$, the partition function, eq. (1.1), now becomes

$$
Z=\mathrm{e}^{-S\left(U^{(0)}\right)} \int \prod_{x, \mu} \mathrm{d} \alpha_{\mu}(x) \prod_{x} \mathrm{~d} \phi^{+}(x) \mathrm{d} \phi(x) \exp \left\{-S_{2}(\alpha, \phi, B)\right\}
$$

From this we get for the non-vanishing contributions to the effective action [11]

$$
S_{\mathrm{eff}}^{(\xi)}=S\left(U^{(0)}\right)+\left\langle S_{\mathrm{T}}\right\rangle-\frac{1}{2}\left\langle S_{A}^{2}\right\rangle-\frac{1}{2}\left\langle S_{B}^{2}\right\rangle+\frac{1}{2}\left(\left\langle S_{\mathrm{sc}, \mathrm{I}}\right\rangle-\frac{1}{2}\left\langle S_{\mathrm{sc}, \mathrm{I}}^{2}\right\rangle\right)
$$

where

$$
\begin{aligned}
S_{\mathrm{sc}, \mathrm{I}} & =S_{\mathrm{sc}}-S_{\mathrm{free}}, \\
S_{\mathrm{free}} & =\frac{1}{2} a^{3} a_{\tau} \sum_{\substack { x \\
\begin{subarray}{c}{\mu, \nu \\
b{ x \\
\begin{subarray} { c } { \mu , \nu \\
b } }\end{subarray}}\left(\Delta_{\mu} \alpha_{\nu}^{b}\right)\left(\Delta_{\mu} \alpha_{\nu}^{b}\right) .
\end{aligned}
$$

All the expectation values in eq. (2.19) have to be calculated with respect to the free action (2.21). Their computation causes no additional problems compared to the symmetric case $[11,12]$. The factor $\frac{1}{2}$ in front of the expectation values of $S_{\mathrm{sc}, \mathrm{I}}$ takes care of the fact, that in four dimensions the ghost field contribution cancels 
just half the contribution of the scalar fields $\alpha_{\mu}$. Finally we obtain for the effective action

$$
\begin{aligned}
S_{\mathrm{eff}}^{(\xi)}= & \int \mathrm{d}^{4} x \sum_{i, a}\left[\left(F_{i 4}^{a}\right)^{2}+\left(F_{4 i}^{a}\right)^{2}\right]\left(\frac{1}{4 g_{\tau}^{2}}-N\left[\frac { N ^ { 2 } - 1 } { 1 6 N ^ { 2 } } \left\{\frac{1}{3} \xi^{-2} I_{1}(\xi)\right.\right.\right. \\
& \left.+\xi^{-1} I_{5}(\xi)\right\}+\frac{1}{64} I_{2 b}(\xi)-\frac{5}{576} I_{2 a}(\xi)+\frac{1}{256} \xi^{-2} I_{4}(\xi) \\
& \left.\left.-\frac{1}{48} \xi^{-2} I_{6}(\xi)-\frac{1}{192} \xi^{-2} I_{7}(\xi)+\frac{11}{12} \operatorname{DIV}(\xi)\right]\right) \\
& +\int \mathrm{d}^{4} x \sum_{i, j, a}\left(F_{i, j}^{a}\right)^{2}\left(\frac{1}{4 g_{\sigma}^{2}}-N\left[\frac{N^{2}-1}{24 N^{2}} I_{1}(\xi)-\frac{5}{288} I_{2 a}(\xi)\right.\right. \\
& \left.\left.+\frac{1}{48} I_{3}(\xi)+\frac{1}{128} I_{4}(\xi)+\frac{11}{12} \operatorname{DIV}(\xi)\right]\right)
\end{aligned}
$$

The functions $I_{1}(\xi)-I_{7}(\xi)$ are defined by three-dimensional integrals given in appendix $\mathrm{A}$; the integrals $I_{1}(\xi)-I_{4}(\xi)$ converge in the limit $\xi \rightarrow \infty$ to the corresponding integrals $I_{1}-I_{4}$ defined in ref. [14]. Thus in the limit $\xi \rightarrow \infty$, as well as for $\xi=1$, eq. (2.22) gives back the hamiltonian and euclidean versions of the effective action as obtained by A. and P. Hasenfratz [14]. The function $\operatorname{DIV}(\xi)$ is defined by the divergent integral

$$
\operatorname{DIV}(\xi)=(2 \pi)^{-4} \iint_{-\pi / 2}^{\pi / 2} \int^{3} \mathrm{~d}^{3} x \int_{(-\pi / 2) \xi}^{(\pi / 2) \xi} \mathrm{d} x_{4}\left(\sum_{i=1}^{3} \sin ^{2} x_{i}+\xi^{2} \sin ^{2}\left(x_{4} / \xi\right)\right)^{-2}
$$

Applying now the invariance condition (2.8) for the effective action, we get the explicit $\xi$-dependence of the couplings $g_{\tau}(a, \xi)$ and $g_{\sigma}(a, \xi)$ as stated in eq. (2.4), with the functions $c_{\tau}(\xi)$ and $c_{\sigma}(\xi)$ given by

$$
\begin{aligned}
c_{\sigma}(\xi)= & 4 N\left[\frac{N^{2}-1}{24 N^{2}}\left\{I_{1}(\xi)-\frac{3}{4}\right\}-\frac{5}{288} I_{2 a}(\xi)+\frac{1}{48} I_{3}(\xi)\right. \\
& \left.+\frac{1}{128} I_{4}(\xi)+\frac{11}{12} \mathrm{FIN}(\xi)+0.010245\right] \\
c_{\tau}(\xi)= & 4 N\left[\frac{N^{2}-1}{16 N^{2}}\left\{\frac{1}{3} \xi^{-2} I_{1}(\xi)+\xi^{-1} I_{5}(\xi)-\frac{1}{2}\right\}+\frac{1}{64} I_{2 b}(\xi)\right. \\
& -\frac{5}{576} I_{2 a}(\xi)+\frac{1}{256} \xi^{-2} I_{4}(\xi)-\frac{1}{48} \xi^{-2} I_{6}(\xi) \\
& \left.-\frac{1}{192} \xi^{-2} I_{7}(\xi)+\frac{11}{12} \operatorname{FIN}(\xi)+0.010245\right]
\end{aligned}
$$

Here $\operatorname{FIN}(\xi)=\operatorname{DIV}(\xi)-\operatorname{DIV}(1)$ is the finite difference of two divergent integrals. The numerical constants in eq. (2.24) and (2.25) result from an evaluation of the integrals $I_{i}(1)$ appearing in $S_{\text {eff }}^{(\xi=1)}$. Their values are contained in table 1 .

Using eq. (2.6) we can now also calculate the ratio $\Lambda(\xi) / \Lambda_{\mathrm{E}}$. We show this ratio in fig. 1 together with $c_{\sigma}(\xi)$ and $c_{\tau}(\xi)$ as a function of $1 / \xi$ for $N=2,3$. We notice, 
TABLE 1

The integrals $I_{1}(\xi)-I_{7}(\xi)$ and their derivatives for $\xi=1$

\begin{tabular}{llccccccc}
\hline \multicolumn{1}{c}{$x$} & \multicolumn{1}{c}{1} & $2 a$ & $2 b$ & 3 & 4 & 5 & 6 & 7 \\
\hline$I_{x}(1)$ & 0.75 & 0.929600 & 0.119734 & 0.103289 & 0.478934 & 0.25 & 0.206578 & 0.309867 \\
$\left(\partial I_{x} / \partial \xi\right)_{\xi=1}$ & 0.440133 & 0.208546 & 0.190133 & 0.033774 & 0.065778 & 0.309867 & 0.238384 & 0.411188 \\
\hline
\end{tabular}

that for $\xi \rightarrow \infty$ all these quantities approach the values

$$
\begin{aligned}
\Lambda(\infty) / \Lambda_{\mathrm{E}} & = \begin{cases}0.84358, & N=2, \\
0.91048, & N=3 ;\end{cases} \\
c_{\sigma}(\infty) & = \begin{cases}0.09958, & N=2, \\
0.18018, & N=3 ;\end{cases}
\end{aligned}
$$
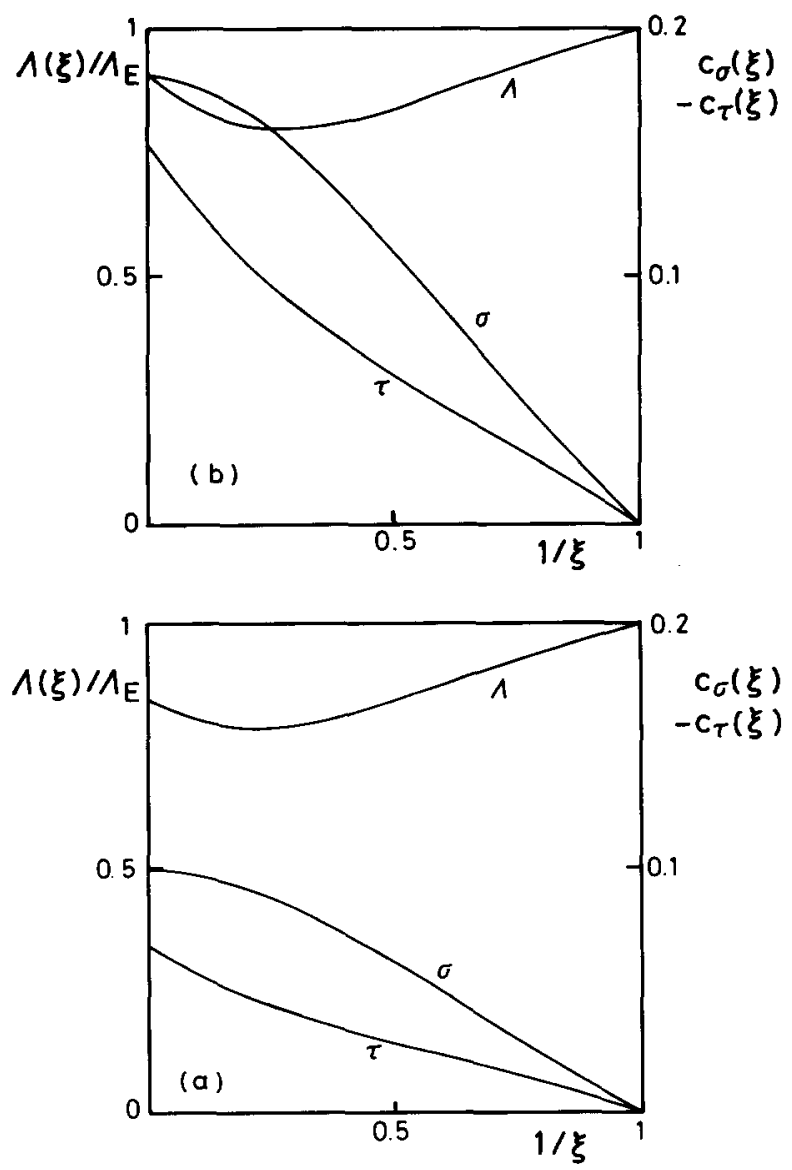

Fig. 1. The functions $c_{\sigma}(\xi)(\sigma),-c_{\tau}(\xi)(\tau)$ and the ratio $A(\xi) / A_{\mathrm{E}}$ of the lattice scale parameters $(\Lambda)$ versus $1 / \xi$ for (a) $N=2$ and (b) $N=3$. 


$$
\mathcal{c}_{\tau}(\infty)= \begin{cases}-0.06798, & N=2, \\ -0.15405, & N=3 ;\end{cases}
$$

obtained in ref. [14]. Finally we calculate the derivatives of the couplings $g_{\sigma}^{-2}(a, \xi)$ and $g_{\tau}^{-2}(a, \xi)$ with respect to $\xi$. As these quantities become relevant in finite temperature Monte Carlo simulations, which have mainly been done on symmetric lattices, we give the result for $\xi=1$ :

$$
\begin{gathered}
\left(\partial g_{\sigma}^{-2} / \partial \xi\right)_{\xi=1}=4 N\left[\frac{N^{2}-1}{32 N^{2}} 0.586844+0.000499\right]= \begin{cases}0.11403, & N=2, \\
0.20161, & N=3 ;\end{cases} \\
\left(\partial g_{\tau}^{-2} / \partial \xi\right)_{\xi=1}=4 N\left[-\frac{N^{2}-1}{32 N^{2}} 0.586844+0.005306\right]= \begin{cases}-0.06759, & N=2, \\
-0.13195, & N=3 .\end{cases}
\end{gathered}
$$

The derivatives of the integrals $I_{1}(\xi)-I_{7}(\xi)$ for $\xi=1$, which are necessary to obtain the numerical values in eqs. $(2.27 \mathrm{a}, \mathrm{b})$ are contained in table 1 . The derivative of $\operatorname{FIN}(\xi)$ yields

$$
(\partial \mathrm{FIN}(\xi) / \partial \xi)_{\xi=1}=0.003166 \text {. }
$$

Notice, that for $\xi=1$ the sum of the derivatives is proportional to $N$; the terms proportional to $\left(N^{2}-1\right) / N$ drop out in this case. Explicitly we have

$$
\left(\partial g_{\sigma}^{-2} / \partial \xi\right)_{\xi=1}+\left(\partial g_{\tau}^{-2} / \partial \xi\right)_{\xi=1}=0.02322 N,
$$

which is identical to $b_{0}$ given in eq. (2.3). How this "sum rule" comes about, we want to discuss in the next section, where we use the invariance of the string tension under changes of $\xi$ to get in the continuum limit eq. (2.29).

\section{Invariance of the string tension}

In the previous section we have determined the dependence of the lattice parameters $g_{\sigma}, g_{\tau}$ and $A$ on the asymmetry factor $\xi$ in the weak coupling limit. We get this dependence from the demand, that in the continuum limit the effective action has to be independent of the lattice cut-off. This is, of course, true for all physical quantities. Thus we can as well use the invariance of any other physical quantity to determine the $\xi$-dependence of the lattice parameters. Using the invariance of the critical temperature of an SU(3)-gluon gas, the derivatives of the couplings $g_{\sigma}, g_{\tau}$ have recently been determined by Monte Carlo simulations [15]. The results agree surprisingly well with our result, eq. (2.27), especially when one bears in mind how difficult it is to get precise values for these numbers by a Monte Carlo simulation.

Here we want to use the invariance of the string tension to explain the "sum rule" (2.29) for the derivatives of the two couplings; we also want to discuss their 
behaviour in the strong coupling regime. On an asymmetric lattice the string tension is defined as

$$
\sigma a_{\mu} a_{\nu}=-\lim _{A \rightarrow \infty} \frac{1}{A} \ln \left\langle W_{\mu \nu}\right\rangle,
$$

where $\left\langle W_{\mu \nu}\right\rangle$ is the expectation value of a planar Wilson loop in the $\mu \nu$-plane of the lattice,

$$
W_{\mu \nu}=\operatorname{tr} \prod_{i \in \partial A} U_{i},
$$

calculated with the partition function (1.1). $A$ is the number of plaquettes enclosed by the boundary $\partial A$ of the Wilson loop.

Let us now consider either pure space-like loops $\left(W_{\mu \nu} \equiv W_{\sigma}\right)$ or space-time loops $\left(W_{\mu \nu} \equiv W_{\tau}\right)$. This gives us two independent conditions for the invariance of $\sigma$ under changes of $\xi$, from which we can determine directly the derivatives of $g_{\sigma}$ and $g_{\tau}$ :

$$
\begin{aligned}
& \partial \sigma a^{2} / \partial \xi=-\lim _{A \rightarrow \infty} \frac{1}{A} \partial \ln \left\langle W_{\sigma}\right\rangle / \partial \xi=0, \\
& \partial \sigma a^{2} / \partial \xi=-\lim _{A \rightarrow \infty} \frac{1}{A} \partial\left(\xi \ln \left\langle W_{\tau}\right\rangle\right) / \partial \xi=0 .
\end{aligned}
$$

Evaluating the derivatives in eqs. $(3.3 \mathrm{a}, \mathrm{b})$ we get a system of two linear equations for $\partial \beta_{\sigma} / \partial \xi$ and $\partial \beta_{\tau} / \partial \xi$ :

$$
\begin{gathered}
\left(\partial \beta_{\sigma} / \partial \xi\right) C_{\sigma \sigma}+\left(\partial \beta_{\tau} / \partial \xi\right) C_{\sigma \tau}=0, \\
\left(\partial \beta_{\sigma} / \partial \xi\right) C_{\tau \sigma}+\left(\partial \beta_{\tau} / \partial \xi\right) C_{\tau \tau}=-\sigma a^{2},
\end{gathered}
$$

where the coefficients $C_{x y}$ are given in terms of plaquette-loops correlation functions as

$$
C_{x y}=\lim _{A \rightarrow \infty} \frac{1}{A\left\langle W_{x}\right\rangle} \sum_{\left\{P_{y}\right\}}\left(\left\langle W_{x} P_{y}\right\rangle-\left\langle W_{x}\right\rangle\left\langle P_{y}\right\rangle\right),
$$

and the sum in eq. (3.5) has to be taken over all " $y$-like" plaquettes. Thus the derivatives of the couplings become

$$
\begin{aligned}
\partial \beta_{\sigma} / \partial \xi & =\left[C_{\sigma \tau} /\left(C_{\sigma \sigma} C_{\tau \tau}-C_{\sigma \tau} C_{\tau \sigma}\right)\right] \sigma a^{2}, \\
\partial \beta_{\tau} / \partial \xi & =-\left[C_{\sigma \sigma} /\left(C_{\sigma \sigma} C_{\tau \tau}-C_{\sigma \tau} C_{\tau \sigma}\right)\right] \sigma a^{2} .
\end{aligned}
$$

For the case $\xi=1$, the eqs. (3.6) simplify, as we have then

$$
C_{\sigma \sigma}=C_{\tau \tau}, \quad C_{\sigma \tau}=C_{\tau \sigma} .
$$

In addition, we notice that on a symmetric lattice the sum of the correlation functions yields

$$
C_{\sigma \sigma}+C_{\sigma \tau}=\partial \sigma a^{2} / \partial g_{\mathrm{E}}^{-2} .
$$


Thus we get an exact expression for the sum of the derivatives of $g_{\sigma}^{-2}$ and $g_{\tau}^{-2}$ at $\xi=1$ :

$$
\left(\partial g_{\sigma}^{-2} / \partial \xi\right)_{\xi=1}+\left(\partial g_{\tau}^{-2} / \partial \xi\right)_{\xi=1}=\frac{-\sigma a^{2}}{\partial \sigma a^{2} / \partial g_{\mathrm{E}}^{-2}}
$$

If we accept the general belief that pure $\mathrm{SU}(N)$ gauge theories are confining for all $N<\infty$, then $\sigma$ stays finite in the continuum limit, while $a$ is connected with $g_{\mathrm{E}}$ through the renormalization group equation (2.2). This yields

$$
\left(\partial g_{\sigma}^{-2} / \partial \xi\right)_{\xi=1}+\left(\partial g_{\tau}^{-2} / \partial \xi\right)_{\xi=1} \underset{g_{E} \rightarrow 0}{=} b_{0}
$$

which is in complete agreement with the result we got from the invariance of the effective action in an entirely different way. This, of course, only indicates that in the weak coupling regime there exists a universal relation between the asymmetry factor $\xi$ and the couplings, such that all physical quantities are invariant under changes of $\xi$, provided one changes also the couplings properly. This is not true in the strong coupling regime. As all physical quantities have different strong coupling expansions, it is impossible to adjust the couplings such, that all of them remain simultaneously invariant under changes of $\xi$. Nevertheless, we have calculated $\left(\partial g_{\sigma(\tau)}^{-2} / \partial \xi\right)_{\xi=1}$ also in the strong coupling regime from the invariance condition $(3.3 \mathrm{a}, \mathrm{b})$ for the string tension, in order to get an idea, how these derivatives behave for large couplings, and for which values the weak coupling relations $(2.27 \mathrm{a}, \mathrm{b})$ become valid. This is particularly useful for Monte Carlo simulations, since these are generally carried out in a region where the couplings are far from being small compared to unity.

In appendix B we give a 12 th order strong coupling expansion of the SU(2) string tension on asymmetric lattices. It is obtained using the graphs for the SU(2) string tension on symmetric lattices as listed in ref. [16]. Taking derivatives with respect to $\xi$ as stated in eq. (3.3) we get a strong coupling expansion for the coefficients $C_{x y}$ contained in the final result, eq. (3.6).

In fig. 2 we display the derivatives $\left(\partial g_{\sigma}^{-2} / \partial \xi\right)_{\xi=1}$ and $\left(\partial g_{\tau}^{-2} / \partial \xi\right)_{\xi=1}$ we obtained from eq. (3.6) by using 10 th and 12 th order strong coupling expansions for the coefficients $C_{x y}$ and the string tension $\sigma a^{2}$ [16]. The expansions cross the weak coupling results at $4 / g_{\mathrm{E}}^{2} \simeq 2.2$; they both diverge for $4 / g_{\mathrm{E}}^{2} \simeq 2.3$, as there $C_{\sigma \sigma}=C_{\tau \tau}$. This seems to indicate that the weak coupling results for $\left(\partial g_{\sigma(\tau)}^{-2} / \partial \xi\right)_{\xi=1}$ can be used in the same region, where the renormalization group relation $(2.2)$ for $g_{\mathrm{E}}(a)$ is valid.

\section{Conclusions}

We have analysed how the $\mathrm{SU}(N)$ gauge theory couplings $g_{\sigma}$ and $g_{\tau}$ as well as the lattice scale parameter $A$, have to be adjusted under changes of the lattice cut-off on asymmetric lattices, in order to leave physical quantities invariant under 


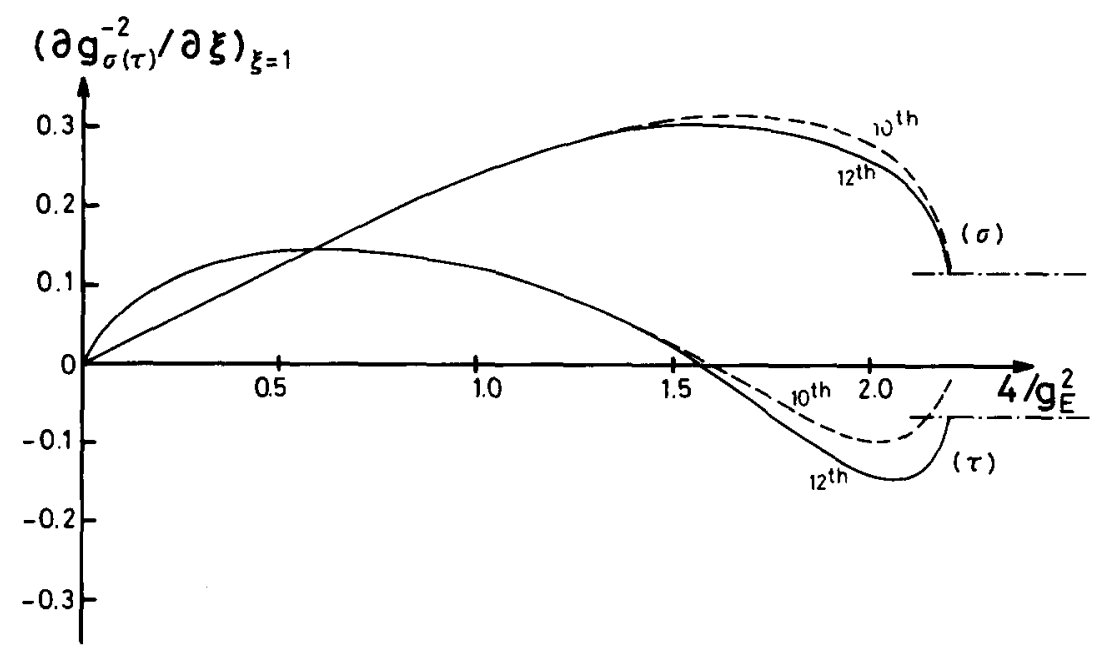

Fig. 2. Derivatives of the couplings $g_{\sigma}^{-2}$ and $g_{\tau}^{-2}$ with respect to $\xi$ for $\xi=1$ from 10 th (dashed curve) and 12 th (full curve) order strong coupling expansion of the $\mathrm{SU}(2)$ string tension versus $4 / g_{\mathrm{E}}^{2}$. Included are also the weak coupling results (dot-dashed curve).

such changes. We have found in the weak coupling limit, that in the whole range $1 \leqslant \xi \leqslant \infty$ of the asymmetry factor $\xi$, the quantum corrections on the couplings $g_{\sigma}^{-2}$, $g_{\tau}^{-2}$ are small. Thus for SU(2), the second "hamiltonian" coupling $\eta=\sqrt{g_{\tau}^{2} / g_{\sigma}^{2}}$ deviates from unity by less than $3 \%$ for $g_{E}^{2} \leqslant 2$. This may justify the approximation $\eta=1$ made in finite-temperature Monte Carlo simulations [7]. However, despite this small effect on the couplings, the quantum corrections give rise to comparatively large effects in the ratio of scale parameters $[\sim 20 \%$ for $\mathrm{SU}(2)]$, and this should be considered in finite-temperature Monte Carlo simulations [7]. These corrections also contribute $\sim 20 \%$ to the derivatives of $\beta_{\sigma}=\left(1 / g_{\sigma}^{2}\right) \xi^{-1}$ and $\beta_{\tau}=\left(1 / g_{\tau}^{2}\right) \xi$, when $g_{\mathrm{E}}^{2} \approx 2$; but in the high-temperature limit [8], when $g_{\mathrm{E}}^{-2} \rightarrow \infty$, this becomes unimportant.

I would like to thank Prof. H. Satz for many discussions and continuous support of this work. I am also grateful to Prof. J. Engels for numerous discussions and his help in the numerical calculations. I appreciate discussions with J.M. Alberty and R. Trinchero.

\section{Appendix A}

THE INTEGRALS $I_{1}(\xi)-I_{7}(\xi)$

We want to give here the definition of the integrals $I_{1}(\xi)-I_{7}(\xi)$ appearing in the effective action, eq. (2.22). All these integrals are three-dimensional integrals, each ranging from 0 to $\frac{1}{2} \pi$. The factors of $\xi$ are arranged such, that the integrals stay 
finite in the limit $\xi \rightarrow \infty$. Using the abbreviation

$$
b^{2}=\sum_{i=1}^{3} \sin ^{2} x_{i}
$$

we have

$$
\begin{aligned}
& I_{1}(\xi)=\xi\left(\frac{2}{\pi}\right)^{3} \int \mathrm{d}^{3} x b\left(\xi^{2}+b^{2}\right)^{-1 / 2}, \\
& I_{2 a}(\xi)=\xi\left(\frac{2}{\pi}\right)^{3} \int \mathrm{d}^{3} x\left(\xi^{2}+2 b^{2}\right) b^{-1}\left(\xi^{2}+b^{2}\right)^{-3 / 2}, \\
& I_{2 b}(\xi)=\xi^{3}\left(\frac{2}{\pi}\right)^{3} \int \mathrm{d}^{3} x\left[b\left(\xi^{2}+b^{2}\right)^{1 / 2}\left(b+\left(\xi^{2}+b^{2}\right)^{1 / 2}\right)^{2}\right]^{-1}, \\
& I_{3}(\xi)=\xi\left(\frac{2}{\pi}\right)^{3} \int \mathrm{d}^{3} x \sin ^{2} x_{1} \sin ^{2} x_{2} \frac{\xi^{2}+2 b^{2}}{b^{3}\left(\xi^{2}+b^{2}\right)^{3 / 2}} \\
& I_{4}(\xi)=\xi\left(\frac{2}{\pi}\right)^{3} \int \mathrm{d}^{3} x \sin ^{2} 2 x_{1} \frac{\xi^{2}+2 b^{2}}{b^{3}\left(\xi^{2}+b^{2}\right)^{3 / 2}}, \\
& I_{5}(\xi)=\xi^{2}\left(\frac{2}{\pi}\right)^{3} \int \mathrm{d}^{3} x\left(\xi^{2}+b^{2}\right)^{-1 / 2}\left(b+\left(\xi^{2}+b^{2}\right)^{1 / 2}\right)^{-1}, \\
& I_{6}(\xi)=\xi^{3}\left(\frac{2}{\pi}\right)^{3} \int \mathrm{d}^{3} x b^{-1}\left(\xi^{2}+b^{2}\right)^{-3 / 2} \cos ^{2} x_{1}, \\
& I_{7}(\xi)=\xi^{3}\left(\frac{2}{\pi}\right)^{3} \int \mathrm{d}^{3} x b^{-1}\left(\xi^{2}+b^{2}\right)^{-3 / 2} .
\end{aligned}
$$

\section{Appendix B}

STRONG COUPLING EXPANSION FOR THE STRING TENSION ON ASYMMETRIC LATTICES

We want to give here the strong coupling expansion for the SU(2) string tension on asymmetric lattices, which is used in sect. 3 to calculate the derivatives of the couplings $g_{\sigma}^{-2}$ and $g_{\tau}^{-2}$ with respect to $\xi$. We have used the graphs given in ref. [16] for the expansion of the string tension on symmetric lattices. On an asymmetric lattice with lattice spacing $a$ in space directions and $a_{\tau}$ in time direction one can derive the string tension either from space like Wilson loops $\left(\sigma_{1} a^{2}\right)$ or from Wilson loops with one space and time direction $\left(\sigma_{\mathrm{II}} a a_{\tau}\right)$,

$$
\sigma_{\mathrm{I}} a^{2}=-\lim _{A \rightarrow \infty} \frac{1}{A} \ln \left\langle W_{\sigma}\right\rangle
$$


TABle 2

The expansion coefficients for the $\mathrm{SU}(2)$ string tension on asymmetric lattices

\begin{tabular}{|c|c|c|c|c|c|c|c|c|}
\hline Order & $\alpha_{1}$ & $\alpha_{2}$ & $\alpha_{3}$ & $\alpha_{4}$ & $\alpha_{5}$ & $\alpha_{6}$ & $c_{\mathbf{I}, \alpha}$ & $c_{\mathrm{II}, \alpha}$ \\
\hline \multirow[t]{3}{*}{4} & 4 & & & & & & 2 & \\
\hline & 2 & 2 & & & & & & 4 \\
\hline & & 4 & & & & & 2 & \\
\hline \multirow[t]{7}{*}{6} & 6 & & & & & & -4 & \\
\hline & 4 & 2 & & & & & & 4 \\
\hline & 4 & & 1 & & & & 6 & \\
\hline & 2 & 4 & & & & & -8 & -12 \\
\hline & 2 & 2 & & 1 & & & & 12 \\
\hline & & 6 & & & & & 4 & \\
\hline & & 4 & 1 & & & & 6 & \\
\hline \multirow[t]{5}{*}{8} & 8 & & & & & & 12 & \\
\hline & 6 & 2 & & & & & & 20 \\
\hline & 4 & 4 & & & & & 16 & 32 \\
\hline & 2 & 6 & & & & & 16 & 4 \\
\hline & & 8 & & & & & 12 & \\
\hline \multirow[t]{23}{*}{10} & 10 & & & & & & -8 & \\
\hline & 8 & 2 & & & & & & -28 \\
\hline & 8 & & 1 & & & & -12 & \\
\hline & 6 & 4 & & & & & -108 & 84 \\
\hline & 6 & 2 & 1 & & & & & 48 \\
\hline & 6 & 2 & & 1 & & & & 48 \\
\hline & 6 & & 2 & & & & 36 & \\
\hline & 4 & 6 & & & & & 4 & -164 \\
\hline & 4 & 4 & 1 & & & & 96 & \\
\hline & 4 & 4 & & 1 & & & & 72 \\
\hline & 4 & 2 & & 2 & & & & 36 \\
\hline & 2 & 8 & & & & & -44 & -12 \\
\hline & 2 & 6 & 1 & & & & 48 & \\
\hline & 2 & 6 & & 1 & & & 48 & \\
\hline & 2 & 4 & 1 & 1 & & & & 24 \\
\hline & 2 & 4 & & 2 & & & & 12 \\
\hline & & 10 & & & & & 36 & \\
\hline & & 8 & 1 & & & & -12 & \\
\hline & & 6 & 2 & & & & 12 & \\
\hline & & 6 & 1 & 1 & & & 24 & \\
\hline & & & 5 & & & & 6 & \\
\hline & & & 2 & 3 & & & & 12 \\
\hline & & & 1 & 4 & & & 6 & \\
\hline \multirow[t]{9}{*}{12} & 12 & & & & & & $\frac{719}{3}$ & \\
\hline & 10 & 2 & & & & & & 36 \\
\hline & 10 & & 1 & & & & -216 & \\
\hline & 9 & & & & & 1 & 8 & \\
\hline & 8 & 4 & & & & & 445 & 624 \\
\hline & 8 & 2 & 1 & & & & & 96 \\
\hline & 8 & 2 & & 1 & & & & -192 \\
\hline & 8 & & 2 & & & & 54 & \\
\hline & 6 & 6 & & & & & 16 & $\frac{508}{3}$ \\
\hline
\end{tabular}


TABLE 2 (continued)

\begin{tabular}{|c|c|c|c|c|c|c|c|c|}
\hline Order & $\alpha_{1}$ & $\alpha_{2}$ & $\alpha_{3}$ & $\alpha_{4}$ & $\alpha_{5}$ & $\alpha_{6}$ & $c_{\mathbf{I}, \alpha}$ & $c_{1 \mathrm{II}, \boldsymbol{\alpha}}$ \\
\hline & 6 & 4 & 1 & & & & -420 & -144 \\
\hline & 6 & 4 & & 1 & & & & 192 \\
\hline & 6 & 2 & 1 & 1 & & & & 144 \\
\hline & 6 & 2 & & 2 & & & & 24 \\
\hline & 6 & & 3 & & & & 24 & \\
\hline & 5 & 4 & & & 1 & & 32 & \\
\hline & 4 & 8 & & & & & 773 & 744 \\
\hline & 4 & 6 & 1 & & & & 204 & \\
\hline & 4 & 6 & & 1 & & & -240 & -792 \\
\hline & 4 & 5 & & & & 1 & & 48 \\
\hline & 4 & 4 & 2 & & & & 144 & \\
\hline & 4 & 4 & & 2 & & & & 156 \\
\hline & 4 & 4 & 1 & 1 & & & & 48 \\
\hline & 4 & 2 & & 3 & & & & 24 \\
\hline & 4 & & 4 & & & & 24 & \\
\hline & 2 & 10 & & & & & -24 & \\
\hline & 2 & 8 & 1 & & & & -300 & -48 \\
\hline & 2 & 8 & & 1 & & & 144 & \\
\hline & 2 & 6 & 1 & 1 & & & 144 & 24 \\
\hline & 2 & 4 & 1 & 2 & & & & 24 \\
\hline & 2 & 2 & 2 & 2 & & & & 24 \\
\hline & 2 & 2 & 1 & 3 & & & & 24 \\
\hline & 1 & 8 & & & 1 & & 8 & \\
\hline & & 12 & & & & & $\frac{383}{3}$ & \\
\hline & & 10 & 1 & & & & -60 & \\
\hline & & 8 & 2 & & & & -18 & \\
\hline & & 8 & 1 & 1 & & & 72 & \\
\hline & & 6 & 2 & 1 & & & 24 & \\
\hline & & 4 & 1 & 3 & & & 24 & \\
\hline & & & 6 & & & & -18 & \\
\hline & & & 2 & 4 & & & -18 & -36 \\
\hline & & -1 & 2 & 3 & & 1 & & 24 \\
\hline & -1 & & 5 & & 1 & & 12 & \\
\hline & -1 & & 1 & 4 & 1 & & 12 & \\
\hline
\end{tabular}

$$
\sigma_{\mathrm{II}} a a_{\tau}=-\lim _{A \rightarrow \infty} \frac{1}{A} \ln \left\langle W_{\tau}\right\rangle
$$

The strong coupling expansions can be given in terms of the expansion parameters $u(x)=I_{2}(x) / I_{1}(x), v(x)=I_{3}(x) / I_{1}(x)$ and $w(x)=I_{4}(x) / I_{1}(x)$, where $I_{i}(x)$ are modified Bessel functions, as

$$
\begin{gathered}
\sigma_{\mathrm{I}} a^{2}=-\ln u\left(\beta_{\sigma}\right)-\sum_{\alpha} c_{\mathrm{I}, \alpha} u\left(\beta_{\sigma}\right)^{\alpha_{1}} u\left(\beta_{\tau}\right)^{\alpha_{2}} v\left(\beta_{\sigma}\right)^{\alpha_{3}} v\left(\beta_{\tau}\right)^{\alpha_{4}} w\left(\beta_{\sigma}\right)^{\alpha_{5}} w\left(\beta_{\tau}\right)^{\alpha_{6}}, \\
\sigma_{\mathrm{II}} a a_{\tau}=-\ln u\left(\beta_{\tau}\right)-\sum_{\alpha} c_{\mathrm{II}, \alpha} u\left(\beta_{\sigma}\right)^{\alpha_{1}} u\left(\beta_{\tau}\right)^{\alpha_{2}} v\left(\beta_{\sigma}\right)^{\alpha_{3}} v\left(\beta_{\tau}\right)^{\alpha_{4}} w\left(\beta_{\sigma}\right)^{\alpha_{5}} w\left(\beta_{\tau}\right)^{\alpha_{6}},
\end{gathered}
$$


with $\beta_{\sigma}=\left(4 / g_{\sigma}^{2}\right) \xi^{-1}$ and $\beta_{\tau}=\left(4 / g_{\tau}^{2}\right) \xi$. The combinatorical factors $c_{\mathrm{I}, \alpha}$ and $c_{\mathrm{II}, \alpha}$ as well as the exponents $\alpha_{1}, \ldots, \alpha_{6}$ contributing up to 12 th order are listed in table 2 .

\section{References}

[1] M. Creutz, Phys. Rev. D21 (1980) 2308

[2] E. Pietarinen, Nucl. Phys. B190 [FS3] (1981) 349

[3] G. Bhanot and C. Rebbi, Nucl. Phys. B180 [FS2] (1981) 469

[4] B. Berg, Phys. Lett. 97B (1980) 401

[5] J. Engels, F. Karsch, I. Montvay and H. Satz, Phys. Lett. 102B (1981) 332

[6] L.D. McLerran and B. Svetitsky, Phys. Lett. 98B (1981) 195

[7] J. Kuti, J. Polónyi and K. Szlachányi, Phys. Lett. 98B (1981) 199

[8] J. Engels, F. Karsch, I. Montvay and H. Satz, Phys. Lett. 101B (1981) 89; Gauge field thermodynamics for SU(2) Yang-Mills systems, Bielefeld preprint BI-TP 81/29 (December, 1981)

[9] K. Kajantie, C. Montonen and E. Pietarinen, Z. Phys. C9 (1981) 253

[10] A. Hasenfratz and P. Hasenfratz, Phys. Lett. 93B (1980) 165

[11] R. Dashen and D.J. Gross, Phys. Rev. D23 (1981) 2340

[12] A. Gonzalez-Arroyo and C.P. Korthals-Altes, Asymptotic freedom scales for any lattice action, Marseille preprint CPT-81/P.1303 (May, 1981)

[13] H. Kawai, R. Nakayama and K. Seo, Nucl. Phys. B189 (1981) 40;

H.S. Sharatchandra, H.J. Thun and P. Weisz, Nucl. Phys. B192 (1981) 205

[14] A. Hasenfratz and P. Hasenfratz, Nucl. Phys. B193 (1981) 210

[15] I. Montvay and E. Pietarinen, Stefan-Boltzmann law at high temperature for the gluon gas, DESY preprint, DESY 81-077 (November, 1981)

[16] G. Münster, Nucl. Phys. B180 [FS2] (1981) 23 